\title{
Uniportal-VATS approach to thoracic endometriosis syndrome: a case report
}

\author{
Gian Maria Ferretti ${ }^{1,2 \#}$, Elisa Meacci ${ }^{1,2 \#}$, Elizabeth Katherine Anna Triumbari, ${ }^{3,4}$ Dania Nachira ${ }^{1,2}$, \\ Maria Teresa Congedo ${ }^{1,2}$, Luca Pogliani ${ }^{1,2}$, Edoardo Zanfrini ${ }^{1,2}$, Amedeo Giuseppe Iaffalfano ${ }^{1,2}$, \\ Leonardo Petracca Ciavarella ${ }^{1,2}$, Maria Letizia Vita ${ }^{1,2}$, Marco Chiappetta ${ }^{1,2}$, Venanzio Porziella ${ }^{1,2}$, \\ Stefano Margaritora ${ }^{1,2}$
}

${ }^{1}$ Dipartimento delle Scienze Cardiovascolari e Toraciche, UOC di Chirurgia Toracica, Fondazione Policlinico Universitario A. Gemelli IRCCS, Rome, Italy; ${ }^{2}$ Istituto di Patologia Speciale Chirurgica, Università Cattolica del Sacro Cuore, Rome, Italy; ${ }^{3}$ Unità di Medicina Nucleare, Fondazione Policlinico Universitario A. Gemelli IRCCS, Rome, Italy; ${ }^{4}$ Istituto di Medicina Nucleare, Università Cattolica del Sacro Cuore, Rome, Italy Contributions: (I) Conception and design: GM Ferretti, E Meacci, S Margaritora; (II) Administrative support: MT Congedo, ML Vita; (III) Provision of study materials or patients: M Chiappetta, V Porziella; (IV) Collection and assembly of data: E Zanfrini, AG Iaffaldano, L Pogliani, D Nachira, LP Ciavarella; (V) Data analysis and interpretation: EKA Triumbari; (VI) Manuscript writing: All authors; (VII) Final approval of manuscript: All authors. \#These authors contributed equally to this work.

Correspondence to: Gian Maria Ferretti, MD. Dipartimento delle Scienze Cardiovascolari e Toraciche, UOC di Chirurgia Toracica, Fondazione Policlinico Universitario A. Gemelli IRCCS, Largo A. Gemelli 1, 00168 Rome, Italy. Email: gianmaria.ferretti86@gmail.com.

\begin{abstract}
Nowadays, endometriosis is considered a common benign disease. It affects between $6 \%$ and $10 \%$ of women of childbearing and it's characterized by the presence of islands of endometrial-like cells outside the uterine cavity, more often located on the intraperitoneal surface of reproductive organs. The thoracic cavity is the second major district where it's possible to find islands of endometriosis affecting the parietal and/or visceral pleural surface, the diaphragm or the lung parenchyma, therefore causing a variety of symptoms and manifestations, all together named as thoracic endometriosis syndrome (TES). The first line therapy for TES is medical aimed at suppressing ovarian steroid hormone production. Indications for pulmonary surgery as a second line therapeutic management of patients with TES are hormonal therapy failure, intolerable adverse effects or symptom recurrence after cessation of medical treatment. Surgery should be the preferred therapeutic option when the patient is planning future pregnancies. To date, videoassisted-thoracoscopy (VATS) represents an important surgical tool in TES patients, as it allows to carry out many procedures for diagnostic and therapeutic purposes. In patients with centrally located bronchial endometriosis, sub-segmentectomies, segmentectomies or lobectomies may be required. We present the case of a patient with TES, treated with a feasible, safe and least invasive thoracic surgical technique available nowadays in many settings: uniportal-VATS.
\end{abstract}

Keywords: Endometriosis; thoracic endometriosis syndrome (TES); uniportal-video-assisted-thoracoscopy (uniportal-VATS)

Received: 13 September 2019; Accepted: 23 September 2019; Published: 15 October 2019.

doi: $10.21037 /$ shc.2019.09.05

View this article at: http://dx.doi.org/10.21037/shc.2019.09.05

\section{Introduction}

Endometriosis is recognized today as being a common benign disease characterized by the presence of islands of endometrial-like cells outside the uterine cavity, more often located on the intraperitoneal surface of reproductive organs. It is estimated that between $6 \%$ and $10 \%$ of women of childbearing age have endometriosis (1). The most frequent manifestations are pelvic pain (about $50-60 \%$ of cases) and infertility (up to $50 \%$ of cases). About $12 \%$ of patients suffering from this disease also present islands of 
endometriosis on organs or viscera of the abdominal cavity not belonging to the female reproductive system $(2,3)$.

The pleural cavity is the second major district where it's possible to find islands of endometriosis. This location in particular may cause catamenial pneumothorax, which is defined as air leaking into the pleural space occurring in conjunction with menstrual periods (catamenial).

Pathogenesis of thoracic endometriosis remains elusive and several theories have been proposed. Surgical findings of associated diaphragmatic endometriosis in case of pleural endometriosis suggest the theory of "retrograde menstruation", with transdiaphragmatic passage and subsequent implantation of the stromal and glandular elements of the endometrium in the thoracic cavity (4).

In 1998, Kirschner et al. (5) introduced the concept of the "porous diaphragm syndrome", suggesting that preexisting diaphragmatic defects may allow gases and fluids to pass through this anatomic boundary. Accordingly, Alifano et al. (6), evaluating the incidence of catamenial pneumothorax among women candidate to surgical treatment for spontaneous pneumothorax, showed that catamenial pneumothorax is more frequent than expected and that diaphragmatic abnormalities seem to play a fundamental role in its pathogenesis.

In the thoracic cavity, islands of endometriosis can be found on the parietal and/or visceral pleural surface, on the diaphragm or within the lung parenchyma. Their localization on different structures and tissues of the pleural cavity allows a variety of symptoms and manifestations, all together named as thoracic endometriosis syndrome (TES).

Among these events there are:

- Catamenial pneumothorax (right sided in $92 \%$ of cases), in $80 \%$ of patients with TES;

* Catamenial haemothorax, $14 \%$ of TES;

* Catamenial haemoptysis, $5 \%$ of TES;

* Lung nodules;

* Catamenial chest pain (caused by intercostal and phrenic nerve irritation);

* Endometriosis-related diaphragmatic hernia.

While the peak incidence of pelvic endometriosis occurs in patients between 24 and 29 years old, the peak incidence for TES is approximately 5 years later and while there is a temporal association between the onset of menstruation and catamenial pneumothorax (72 hours from the beginning of menstruations), this is not equally true with the rest of the manifestations of TES (7).

TES' chronologically related presentation with menstruation, its mainly right-sided symptoms (in about
$88 \%$ of cases), young patient's age, characteristics of recurrence and a history of infertility are features that permit an easier and more rapid differential diagnosis between TES and many other thoracic symptomatic pathologies (8).

Diagnosis is therefore based on a thorough medical history and on radiological and laboratory tests. Chest radiography is the simplest and least expensive test to confirm the presence of pneumothorax and pleural effusion. A chest computed tomography (CT) scan is the gold-standard diagnostic tool for the detection of pneumomediastinum, pneumoperitoneum, ground glass opacities, bronchial wall thickening, thin-wall cavities within the lung parenchyma, bullous formations.

The use of magnetic resonance (MR) imaging is generally limited to the study of diaphragmatic localizations of endometriosis and related hernias (9).

The first line management behavior for TES is medical treatment: gonadotropin-releasing hormone $(\mathrm{GnRH})$ analogs, GnRH antagonists, progestins, oral contraceptives, danazol, aromatase inhibitors. The goal is to suppress ovarian steroid hormone production, which also potentially reduces the risk of recurrence (10).

Indications for pulmonary surgery as a second line therapeutic management of patients with TES are hormonal therapy failure, intolerable adverse effects or symptom recurrence after cessation of medical treatment. Surgery should be the preferred therapeutic option when the patient is planning future pregnancies.

We present the case of a patient with TES, treated with the less invasive thoracic surgical technique available nowadays.

\section{Case presentation}

A 24-year-old woman presented at our emergency room after a 2-month history of intermittent, sharp, pleuritic chest pain radiating from the right subscapular region, onset of productive white-sputum cough and progressively worsening dyspnea that limited her activities till persisting at rest.

The patient had no history of trauma and denied having had fever, rash, joint pain, nausea, hemoptysis, orthopnea, hematochezia, dizziness or visual changes.

During anamnesis, no history of tuberculosis exposures was detected, and a recent tuberculin skin test was reported as negative.

She was an active smoker of 10 cigarettes per day, 


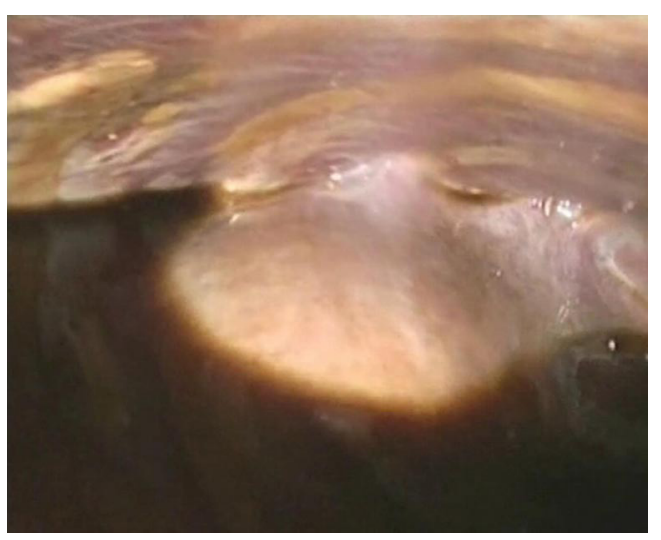

Figure 1 Pleural cavity with the presence of a chocolate-brown fluid effusion.

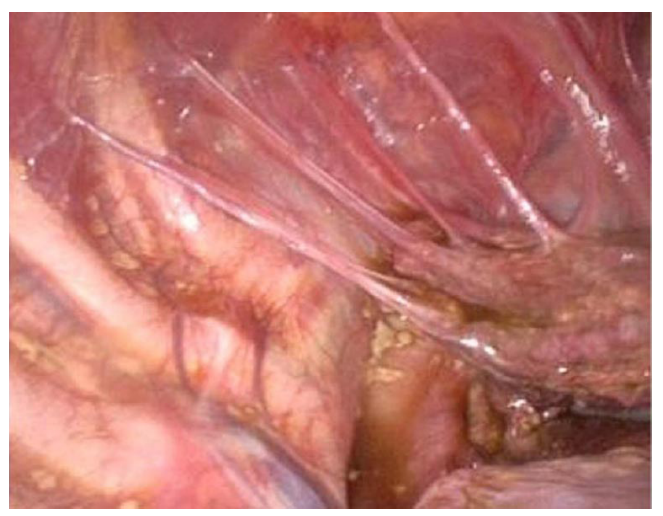

Figure 2 Adhesions between lung and parietal pleura probably caused by post-inflammatory pleuritis caused by repeated unrecognized episodes of pleural effusion and pneumothorax.

reported social use of alcohol and denied assumption of recreational drugs.

Physical examination of the chest revealed decreased vocal resonance and fremitus on the right hemithorax.

Laboratory tests were within normal ranges. An electrocardiography showed a sinus tachycardia. A chest $\mathrm{X}$-ray showed a significant right-sided pleural effusion with an apical pneumothorax.

The subsequent thoracentesis revealed a pleural blood effusion.

The patient was therefore referred to our Thoracic Surgery Department for further tests and differential diagnosis of pathologies presenting with pleural blood effusion in young women was considered: malignancy (primary or metastatic), pulmonary embolism, serositis, pancreatitis, thoracic aneurysm, Meigs' syndrome, TES (11).

Levels of coagulation factors, $\mathrm{C}$ protein and antithrombin factor III (AT-III) were normal. Cancer antigen 15-3 (CA 15-3), cancer antigen 50 (CA 50), carcinoembryonic antigen (CEA), carbohydrate antigen 19-9 (CA 19-9), neuronspecific enolase (NSE), cancer antigen 125 (CA-125), alphafetoprotein (AFP), beta human chorionic gonadotropin (BhCG) and tissue plasminogen activator (TPA) levels were normal. Pleural effusion culture results and cytological examination were negative.

The patient denied being on any medications, but she had discontinued estrogen-progestin treatment two months before, after ten years of administration. Menses were regular and she denied gynecologic complaints, pelvic pain or dyspareunia. The patient was in her second day of menstruation.

Breast, abdomen and pelvic ultrasonography were negative.

A chest CT-scan confirmed a right-sided pleural effusion with a blood-density aspect, pleural thickening, a small bleb in the middle lobe and air in the right apical region.

As the suspect of an ongoing TES was strong, a videothoracoscopy was performed with both a diagnostic and therapeutic purpose.

The procedure was performed under general anesthesia and single lung ventilation, using a double-lumen endotracheal tube. The patient was then placed in right lateral decubitus position. A single 4-cm muscle-sparing incision [uniportal-video-assisted-thoracoscopy (uniportalVATS)] was performed at the right 5 th intercostal space on the mid axillary line. Incision widening and protection for the introduction of a $10 \mathrm{~mm} 30^{\circ}$ thoracoscope and endoscopic instruments was obtained through a wound protector $(12,13)$.

Exploration of the pleural cavity revealed the presence of a chocolate-brown fluid (Figure 1) and strong adhesions between diaphragm, lung and mediastinum, probably caused by post-inflammatory pleuritis caused by repeated unrecognized episodes of pleural effusion and pneumothorax (Figure 2).

A complete lysis of adhesions was performed. A dense nodule was then observed on the visceral pleura (Figure 3) and several millimetric perforations associated with purplish nodules were found on the centrum tendineum of the diaphragm (Figure 4). A diaphragmatic resection was therefore performed, and the defect was closed with 4 stiches of prolene 1 interrupted suture.

The hydro-pulmonary re-expansion test documented 


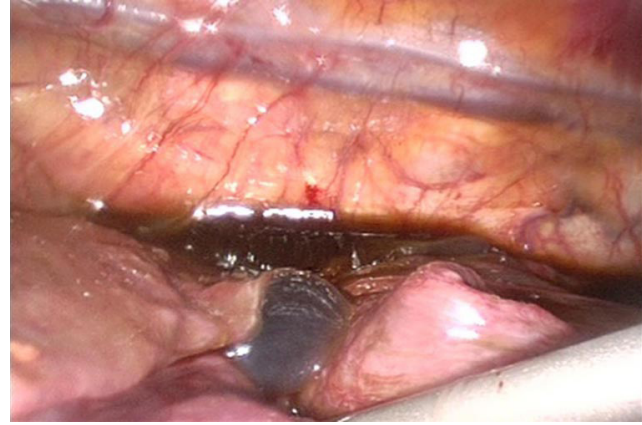

Figure 3 Nodule on the visceral pleural surface, intraoperative finding.

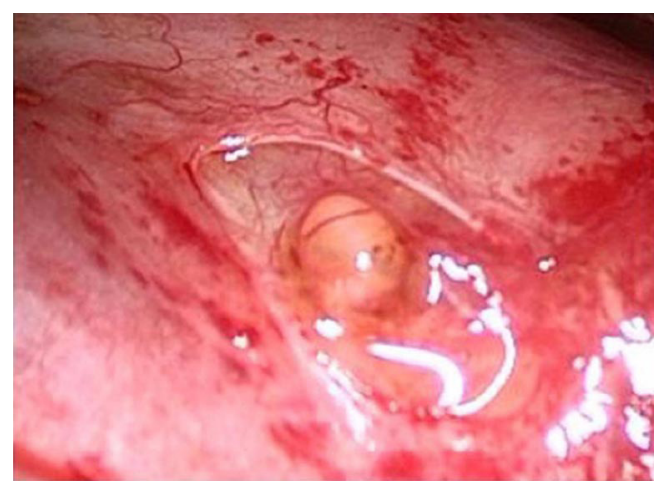

Figure 4 Nodule on the diaphragm, intraoperative finding.

aerial loss from the apex of the lung, where bubbles were present. An apicectomy was hence performed using a mechanical stapler. A chest drainage tube was left in site through the incision.

The post-operative course was uneventful, and the pleural drainage was removed on the fourth post-operative day. The patient was discharged on the next day without complications.

Pathological examination of the resected pleural and diaphragmatic tissue showed an extensive non-malignant organized pleuritis and small foci of glandular tissue resembling endometrial glands and stroma, consistent with endometriosis.

After collegial discussion in a muldisciplinary team including gynecologists, the decision to advise the patient to undergo a laparoscopy was taken, considering the strong association between TES and pelvic endometriosis. Laparoscopic exploration of the abdomen and pelvis confirmed the suspicion of disseminated pelvic endometriosis and allowed the removal of endometrial foci from the peritoneal side of the diaphragm. The previous diaphragmatic suture was found in order.

The patient was discharged on the 4th post-operative day and is currently in follow-up with her gynecologist, with no other reported episodes of recurrence of TES.

\section{Discussion}

To date, VATS represents an important surgical tool in TES patients, as it allows to carry out many procedures for diagnostic and therapeutic purposes.

In peripheral lesions, VATS surgery has been demonstrated to represent the preferred method for the performance of wedge resections, pleurodesis, atypical resections of TES-related pulmonary nodules, bullectomies and removal of pleural nodularities by electrocautery, laser or plasma energy (14-16).

In patients with centrally located bronchial endometriosis, sub-segmentectomies, segmentectomies or lobectomies may be required (17).

Diaphragmatic plastic or resections can also be performed if this muscle has been affected by endometriosis.

In the past few years, the uniportal thoracic surgical technique has demonstrated its feasibility and safety in many settings. Its plasticity allows to carry out surgical therapeutic procedures on all thoracic organs, enabling surgeons to approach all thoracic structures from one single incision during the same surgical intervention. Therefore, uniportal-VATS could become a potential first choice approach in patients with TES, who would benefit from not having to undergo videothoracoscopies and/or open surgery in different timings.

Some challenges are currently still standing. In recent years, women's frequent use of the estrogen-progestin pill-which generates atrophy of the endometrial tissue-has made diagnosis of TES very complicated as symptomatology is lighter and more subtle. Therefore, it is of primary importance to consider TES among differential diagnoses in women of childbearing age presenting with thoracic symptoms and a history of estrogen-progestin pill assumption and to include an extremely accurate inspection of the pleural cavity and thorax anatomical structures during surgical interventions in these patients. To do so, it appears fundamental to be able to apply a VATS approach, that allows a magnified vision and higher diagnostic accuracy.

A last consideration is needed to take into note that the association of TES and pelvic endometriosis is strong. Therefore, the approach to surgical treatment should 
always include multidisciplinary evaluation of the case to consider the possibility to perform both laparoscopy and thoracoscopy during the same surgical session (18).

\section{Acknowledgments}

Funding: None.

\section{Footnote}

Provenance and Peer Review: This article was commissioned by the editorial office, Shanghai Chest for the series "The Second Roman Tips and Tricks in Thoracic Surgery the Great Potential of Uniportal VATS". The article has undergone external peer review.

Conflicts of Interest: All authors have completed the ICMJE uniform disclosure form (available at http://dx.doi. org/10.21037/shc.2019.09.05). The series "The Second Roman Tips and Tricks in Thoracic Surgery - the Great Potential of Uniportal VATS" was commissioned by the editorial office without any funding or sponsorship. EM served as the unpaid Guest Editor of the series and serves as an unpaid editorial board member of Shanghai Chest from Mar 2018 to Feb 2020. SM served as the unpaid Guest Editor of the series. The authors have no other conflicts of interest to declare.

Ethical Statement: The authors are accountable for all aspects of the work in ensuring that questions related to the accuracy or integrity of any part of the work are appropriately investigated and resolved. All procedures performed in studies involving human participants were in accordance with the ethical standards of the institutional and/or national research committee(s) and with the Helsinki Declaration (as revised in 2013). Written informed consent was obtained from the patient for publication of this manuscript and any accompanying images.

Open Access Statement: This is an Open Access article distributed in accordance with the Creative Commons Attribution-NonCommercial-NoDerivs 4.0 International License (CC BY-NC-ND 4.0), which permits the noncommercial replication and distribution of the article with the strict proviso that no changes or edits are made and the original work is properly cited (including links to both the formal publication through the relevant DOI and the license). See: https://creativecommons.org/licenses/by-nc-nd/4.0/.

\section{References}

1. Nezhat C, Li A, Abed S, et al. Strong association between endometriosis and symptomatic leiomyomas. JSLS 2016. doi: 10.4293/JSLS.2016.00053.

2. Giudice LC. Clinical practice. Endometriosis. N Engl J Med 2010;362:2389-98.

3. Joseph J, Sahn SA. Thoracic endometriosis syndrome: new observations from an analysis of 110 cases. Am J Med 1996;100:164-70.

4. Ziedalski TM, Sankaranarayanan V, Chitkara RK. Thoracic endometriosis: a case report and literature review. J Thorac Cardiovasc Surg 2004;127:1513-4.

5. Kirschner PA. Porous diaphragm syndromes. Chest Surg Clin N Am 1998;8:449-72.

6. Alifano M, Trisolini R, Cancellieri A, et al. Thoracic endometriosis: current knowledge. Ann Thorac Surg 2006;81:761-9.

7. Nezhat C, Lindheim SR, Backhus L, et al. Thoracic endometriosis syndrome: a review of diagnosis and management. JSLS 2019. doi: 10.4293/JSLS.2019.00029.

8. Cassina PC, Hauser M, Kacl G, et al. Catamenial hemoptysis. Diagnosis with MRI. Chest 1997;111:1447-50.

9. Rousset P, Rousset-Jablonski C, Alifano M, et al. Thoracic endometriosis syndrome: CT and MRI features. Clin Radiol 2014;69:323-30.

10. Koizumi T, Inagaki H, Takabayashi Y, et al. Successful use of gonadotropin-releasing hormone agonist in a patient with pulmonary endometriosis. Respiration 1999;66:544-6.

11. Black H, Sigal D, Barnes D, et al. A 25-year-old patient with spontaneous hemothorax. Chest 2005;128:3080-1, 3082-3.

12. Ferretti GM, Congedo MT, Pogliani L, et al. Uniportalvideo-assisted thoracoscopic surgery resection of a bronchogenic cyst: a case report. Shanghai Chest 2019;3:40.

13. Ismail $M$, Swierzy $M$, Nachira $D$, et al. Uniportal videoassisted thoracic surgery for major lung resections: pitfalls, tips and tricks. J Thorac Dis 2017;9:885-97.

14. Alifano M, Roth T, Broët SC, et al. Catamenial pneumothorax: a prospective study. Chest 2003;124:1004-8.

15. Corson SL, Woodland M, Frishman G, et al. Treatment of endometriosis with a Nd:YAG tissue-contact laser probe via laparoscopy. Int J Fertil 1989;34:284-8.

16. Marshall MB, Ahmed Z, Kucharczuk JC, et al. Catamenial 
pneumothorax: optimal hormonal and surgical management. Eur J Cardiothorac Surg 2005;27:662-6.

17. Inoue T, Kurokawa Y, Kaiwa Y, et al. Video-assisted thoracoscopic surgery for catamenial hemoptysis. Chest 2001;120:655-8.

doi: $10.21037 /$ shc.2019.09.05

Cite this article as: Ferretti GM, Meacci E, Triumbari EKA, Nachira D, Congedo MT, Pogliani L, Zanfrini E, Iaffalfano AG, Ciavarella LP, Vita ML, Chiappetta M, Porziella V, Margaritora S. Uniportal-VATS approach to thoracic endometriosis syndrome: a case report. Shanghai Chest 2019;3:61.
18. Nezhat C, Nicoll LM, Bhagan L, et al. Endometriosis of the diaphragm: four cases treated with a combination of laparoscopy and thoracoscopy. J Minim Invasive Gynecol 2009; 16:573-80. 